\title{
Research on the Application of AIRO Model in Indirect Economic Loss Assessment of Disaster
}

Peng Zhang, Lizhi Wu, Jing Xin, Jing Liu

Chinese People's Armed Police Forces Academy, Langfang 065000, China

\section{ARIO 模型应用于灾害间接损失评估的拓展性 \\ 研究}

\author{
张鹏, 吴立志, 辛晶, 刘静 \\ 中国人民武装警察部队学院, 河北廊坊 065000 , 中国
}

\begin{abstract}
The assessment of the indirect economic loss cased by the disaster has great significance on the reconstruction. However, when assessing the indirect loss after disaster, it needs research on the scientific and sensitivity of the parameters of the model structure and the availability of the corresponding input-output table. This paper use RAS method for the input-output correction, which make up for the dis-match of time and the defects of the input-output table caused by the indirect economic loss. This paper constructs the LIO table in 1997,1998 and 2002, applies the ARIO model and analyzes the indirect economic loss in floods in 1998. The results show that when assess the post-disaster, the input and output data can be replaced. This paper provides new ideas on the use of input-output model calculating potential indirect economic loss.
\end{abstract}

Key words: Input-Output model; indirect economic loss assessment; RAS ; IO table

摘 要

准确评估灾害对经济冲击造成的间接 经济损失对于政府采取正确的灾后重建策 略具有重要意义。然而在进行灾后间接损失 评估时, 除了要对模型构造的科学性和参数 的敏感性进行研究外, 还需要保障模型构造 所需对应的投入产出表的可获得性。本文采 用 RAS 法用于对投入产出表进行修正，弥 补了使用投入产出法评估灾害间接经济损
失时, 灾害发生时间与所用投入产出表不匹 配的缺陷。本文构造了 1997、1998、2002 年 13 部门的 LIO 表, 并应用 ARIO 模型对 湖南省 1998 年洪涝灾害引发的等量直接经 济损失造成的间接经济损失进行对比分析。 结果表明在进行灾后粗略评估时, 临近年份 投入产出数据具有可替代性。本文为使用投 入产出模型计算灾害潜在间接经济损失拓 宽了思路。

关键字: 投入产出模型; 间接经济损失评估; RAS 法; 投入产出表

1 引入

巨大的灾害不仅带来巨大的人员伤亡 的同时，还造成难以估量的财产损失。随着 区域经济的融合度进一步发展, 世界经济融 为一体, 各地经济的依存度进一步提高。当 巨灾对某一地区经济造成巨大打击的同时， 通过灾害对经济系统冲击的涟渏效益, 会产 生数倍于直接经济损失的间接经济损失 ${ }^{[1]}$ 。 因此对于巨大的灾难事件，不仅从灾害学、 管理学、新闻学的角度之外, 从经济学的视 角对灾害的影响范围对区域和国家水平进 行研究和思考, 将具有更加重要的现实意 义。灾害经济学的概念产生于上世纪 50、60 
Risk Analysis and Crisis Response in Big Data Era (RAC-16)

年代, 随着用经济学的角度对灾害影响进行 研究的逐步深入, 经济学模型作为工具对对 灾害给经济的冲击影响进行研究的学者越 来越多, 而应用可计算一般均衡模型 (computable general equilibrium, CGE) 和投入产出模型 (input-output mode1, I0) 为主的以经济学为基础的评估模型, 逐渐得 到广大相关研究人员的认可。其中 ARIO (Adaptive Regional Input-Output) 模型 在传统 IO 模型的基础上进行了改进用于评 估巨灾造成的间接损失方面, 在国内外已经 得到比较广泛的应用, 国外如 Stephane 研 究了卡特里娜县风对新奥尔良地区的间接 经济损失 ${ }^{[1]}$, 国内的学者如吴吉东、丁先军、 李宁以 2008 年发生在中国四川省汶川地震 为研究对象对灾害造成的间接经济影响进 行了评估 ${ }^{[2-4]}$, 张鹏等以 1998 年湖南省遭遇 的洪涝灾害的重大损失为背景, 对多个部门

2 方法介绍

本文针对的模型扩展性研究首先需要对投 间生产中的直接消耗结构。但由于编制投入 入产出延长表的编制需求和方法进行介绍。

\section{1 投入产出延长表的编制}

在使用当地投入产出表 LIO 时对灾害 造成的间接经济损失进行评估时, 可能会出 现灾害发生年没有编制投入产出表的情况。 通常在模型要求不很严格的情况下, 可以用 灾害发生的临近年代替。比如, 本文就是用 1997 年 IO 表, 评估 1998 年洪涝灾害。除 此之外, 为了更准确评估灾后各部门经济的 具体恢复情况, 就需要编制投入产出延长表 （如 1997 年 IO 表和 2002 年 IO 表之间的投 入产出延长表)。

\subsection{1 修正投入产出表的目的}

对静态投入产出分析来说, 一张投入产
的间接经济损失进行了模拟并对模型使用 中的参数敏感性进行了分析 ${ }^{[5-7]}$ 。此方法也 是作为灾后用 GIS 手段进行评估的补充 ${ }^{[8]}$ 。

ARIO 模型对于灾害给一个区域经济造 成巨大冲击可以进行很好的模拟, 但一个不 可忽视的局限在于, ARIO 模型依赖于灾害 发生时地区投入产出表的获取, 由于投入产 出表的发布周期是 5 年, 因此会出现灾害冲 击造成相同直接经济损失发生在不同年代 造成的间接损失不同的探讨。而不同年代反 应在 ARIO 模型中时, 可以直接理解为不同 的投入产出表代表的不同的经济产业结构。 本文针对具体年份需求更为迫切的模型需 求, 采用 RAS 法对有限的投入产出表进行 修正, 得到所需的新的投入产出表, 此方法 可用于投入产出模型对区域未来间接经济 损失风险进行预测分析时的应用。

出表的直接消耗系数只是反映一个特定时 产出表要花费大量的人力、物力、财力, 所 以绝大部分国家目前都未实现一年编一张 表。而随着经济技术的不断发展，各种投入 消耗系数经常在变动之中, 这样在编制新表 之前, 若一成不变地使用旧表的消耗系数来 表示各年的消耗结构就会产生误差, 需要及 时进行调整和修订。

\subsection{2 投入产出表的修正方法}

投入产出表的修正的主要难度在于修 正投入产出直接消耗系数。而该系数的修正 修正法。全面修正法通过调查各方面的资料 得到一手数据, 按照投入产出表的编制要 求, 重新编制该表同时全面修正直接消耗系 方法根据修正的全面程度, 分为全面和局部 


\section{Risk Analysis and Crisis Response in Big Data Era (RAC-16)}

数; 而局部修正法根据技术、经济、自然等 因素和有关统计资料, 只选择变化较大的直 接消耗系数局部地进行调整的方法。我国与 世界大部分国家一样，一般采用 5 年左右重 新编制投入产出表, 用于指导产业部门的发 展，从总体和宏观上对国家或者地区整体经 济各方面进行分析。而在编制新表期间则采 取局部调整, 通过局部调整得到的投入产出 表一般被称为投入产出延长表。RAS 法就是 一种对直接消耗系数进行局部调整的常用 方法。英国经济计量学家 $\mathrm{R}$.斯通提出的 $R A S$ 法, 也被称为适时修正法。由于篇幅有限, $R A S$ 法修正投入产出表的直接消耗系数的 具体流程请参考文献 ${ }^{[9]}$ 。

\section{2 投入产出表的编制及意义}

根据上述方法修正 1998 年湖南省投入 产出表。首先, 选取 1997 年湖南省投入产 出表为基年投入产出表, 得到基年投入产出 表对应各象限值。其次, 选取目标年投入产 出表除第一象限外其他象限各值, 通过投入 产出表平衡关系得到中间投入和中间产出 的部门合计值。应用 $R A S$ 法多次迭代, 得到 目标年投入产出表。

由此, 本研究按照 $R A S$ 法得到的 1998 年投入产出表的直接消耗系数矩阵, 进而得 到第一象限值, 然后补充第二、第三象限各 部分值得到完整的 1998 年投入产出表。用 于目标年投入产出表编制的统计数据包括 《湖南省统计年鉴 (1999)》中对 1998 年各 部门总产出、增加值、消费、资本形成总额 等数据, 因为湖南省历年统计年鉴中增加值 等数据只给出 13 部门, 同时为便于模型相 关结论的验证, 建立 13 部门 LIO 表。对于 统计年鉴无法获取的数据, 根据投入产出延
长表编制经验, 将与 1997 年投入产出表的 对应部分进行相同口径放大或缩小得到, 对 投入产出表整体影响很小, 编制好的新投入 产出表需要进行部门和总量平衡检查。修正 好的 1998 年投入产出表, 即 1998 年投入产 出延长表。

编制投入产出延长表对于灵活应对特 定时间发生的灾害造成间接经济损失有一 定意义。首先, 是应对恢复期比较短的灾害 的间接损失的评估, 某些灾害造成的直接经 济损失有限, 重建期短, 或是发生在两次投 入产出表中间时, 是造成应用模型评估对应 数据不准确的原因。其次, 为计算未来的灾 害风险做准备, 通过研究投入产出延长表的 编制方法, 配合经济学中其他预测经济发展 的方法, 可对未来 5-10 年的地区经济做预 测, 这样对未来 10 年发生的巨灾事件, 造 成的间接经济损失, 及对各产业部门的影响 等均可进行有益的评估, 为政府提前安排减 灾资金的投入，减灾政策的制定提供参考。

\section{3 实例分析}

在灾害对经济系统造成相同直接损失 情况下, 由于灾害发生在不同年代的经济环 境不同, 投入产出表中可以将这种因为不同 发展阶段的经济结构不同通过投入产出表 的数量经济的平衡关系反映出来。因为经济 系统环境变化造成的间接经济损失不同, 基 于这样原理, 就可以通过编制未来的投入产 出表进行未来的风险预测。本文通过编制 1997 年、 2002 年 13 部门当地投入产出表, 以及 1998 年 13 部门投入产出延长表, 来评 估在模型其他参数不变的情况下, 因社会经 济结构变动对间接经济损失的影响。本文中 投入产出延长表编制方法采用 RAS 法修正 
Risk Analysis and Crisis Response in Big Data Era (RAC-16)

第一象限, 因篇幅有限, 编制完成的投入产 出表此文略。

表 1 对比了 1998 年湖南洪涝灾害使用 1997、1998、2002 年不同LIO（也可以说是 相同强度的灾害发生在不同年代) 的间接经 济损失, 文中重建政策采用产业部门 3 个月 后最大生产能力达到灾前的 $120 \%$ 。

表 1 不同年份的间接损失和恢复期比较

\begin{tabular}{llll}
\hline & $\mathbf{1 9 9 7}$ & $\mathbf{1 9 9 8}$ & $\mathbf{2 0 0 2}$ \\
\hline 总损失/直接损失 & 1.28 & 1.31 & 1.32 \\
间接经济损失值 & 130.72 & 143.32 & 148.04 \\
恢复期 (月) & 35 & 34 & 32 \\
\hline
\end{tabular}

本文恢复期以完成总体重建量的 $99 \%$ 为限, 从表 1 结果看, 重建期相差不大。虽 然各年的经济结构情况不同, 但从总体看经 济发展水平高的年份重建期略短。本文中选 择 1998 和 2002 年作为 1997 年的对比值的 原因, 1998 年为以 1997 年 IO 表为基年用 RAS 法编制出来的投入产出延长表, 可以明 确连续相隔两年的间接经济损失变化范围; 2002 为湖南省统计局编制的投入产出表, 1998 年得到的间接经济损失数值与 1997 年 和 2002 年求出的间接经济损失在增长趋势 上是一致的, 说明用 RAS 法编制投入产出 延长表具有一定的可信度。

间接经济损失如何变化与多种因素有 关，不仅与投入产出表中的各产业部门之间 的投入产出联系有关, 同时与进出口值有 关。为看出仅仅由于产业部门相互投入产出 关系的变化影响的间接损失变化, 因此采用 1997、1998、2002 的LIO做 3 次模拟过程中, 均未根据年份做生产资本的相应调整, 本文 中全部使用 1998 年洪涝灾害时候湖南省的
生产资本累计值。这里未考虑各个年代实际 的生产资本增加值变化的影响因素, 而实际 上中国快速的经济增长加速了资本的积累。 图 1和图 2 为不同年份 $(1997 、 1998 、 2002)$ 的增加值变化率和增加值的比较。

从图 1可以看出, 1997 年和 1998 年增 加值变化率几乎相同, 2002 年与其相比与灾 前增加值相比, 变化率下降幅度稍小, 大约 30 个月之后, 三种增加值变化率模拟情况相 差不大。还可看出, 采用不同年份的LIO 表, 恢复重建期几乎无变化, 而灾区总增加值相 差较大, 总增加值主要体现重建量, 因此可 以看出随着时间推移, 相同灾害强度造成的 间接损失越大, 灾后需要重建的增加值也越 大。在实验中还对不同年份重建需求的变化 量进行了比较, 结果表明 1997、1998、2002 的需求曲线变化不大, 可认为重建发生在不 同年份各阶段重建过程相差不大, 基本在相 同时间段可完成几乎相等的重建量, 重建完 成时间基本一致。

通过 1997 和 1998 年两年的 LIO 表计算 结果可知, 对于评估结果允许一定误差情况 下, 用相邻两年的 IO 表互相代替, 结果相 差不大。对于成熟发达国家或者极端贫穷国 家来说, 社会经济结构在较长的时间内保持 相对稳定, 因此是否考虑恢复期经济结构变 化对重建时间的影响不大, 而中国处于高速 发展时期，国家和企业投资、居民消费激增， 进出口不断增大等因素导致的中国经济结 构变化比其他国家快, 而中国经济又具有相 当规模, 加之中国的特色国情 (救灾的举国 体制), 在恢复重建中考虑经济结构调整更 符合中国实际。 
Risk Analysis and Crisis Response in Big Data Era (RAC-16)

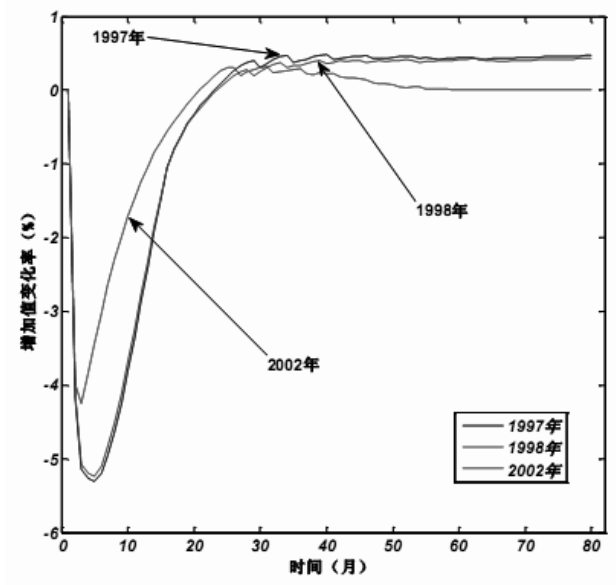

图 1 不同年份的增加值变化率比较

4 讨论

ARIO 模型用以模拟灾后间接经济损 失, 必不可少的要进行前提假设, 最重要的 包括模型构造前提条件以及构造区域投入 产出表时的部门合并，在下文中进行详述。

应用投入产出模型定量计算灾害的产 业关联性间接经济损失, 前提的假设条件如 灾害仅破坏原有的经济系统的均衡状态, 但 不影响经济系统中的产业结构, 而实际上, 巨灾必将导致区域经济结构的改变。一方 面, 巨灾将导致一部分企业经济活动停止; 另一方面, 巨灾将影响到一部分企业生产的 商品的来源或销路渠道改变, 寻找新的代替 厂商。这两方面将逐步改变区域的经济结 构, 随着重建过程推移, 最终区域经济系统 将达到新的平衡。

另外构建 ARIO 模型所需的区域投入产 出表过程中, 由于部门合并的关系, 灾害对 中小企业的影响被忽略, 也会对评估结果产 生一定影响。从另一个角度, 部门数量也可 以看作为区域经济结构的丰富程度, 因为减 少的部门同样可以认为被合并部门是由于

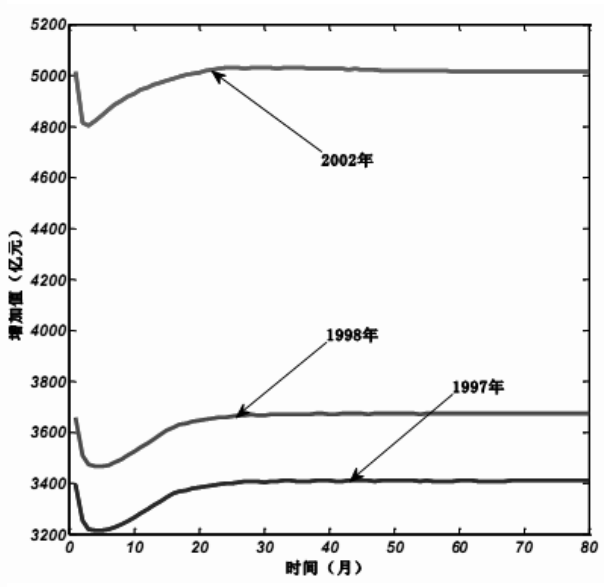

图 2 不同年份的增加值比较

该部门缺乏数据, 试想在一个较小范围地 区, 国民经济中各个部门并非都存在, 那么 这部分数据为空的部门可以认为部门不存 在或者部门产出为 0 。这样将当地 LIO 表中 部门数量与区域经济结构联系起来, 从一个 侧面反映部门数量多的 LIO 经济结构比较 丰富, 发达程度相对较高, 部门数量少的 LIO 经济结构一般, 发达程度较低。

本文仅基于灾后恢复重建的理想假设, 初步对洪涝灾害造成的间接经济损失及重 建期进行评估, 而忽略了一些对间接经济影 响起作用的因素。具体如下为, 真实的恢复 重建受当地及外部经济环境、劳动力供给、 物价水平、长期的财政金融政策等多种因素 的共同作用。而实际灾后经济环境要复杂的 多, 除了投入产出表所能体现的经济要素 外, 由于商品短缺, 导致物价受到影响, 劳 动力短缺造成的产业部门生产能力下降, 进 出口的替代性以及各类经济参数的调整等 未能考虑进去等诸多现实因素的科学考虑, 也是今后需要研究的问题。 
Risk Analysis and Crisis Response in Big Data Era (RAC-16)

5 结论

本文对应用 ARIO 模型评估灾害间接经 济损失的应用方面进行了探索性研究, 对模 型的应用前景进行拓展。首先对模型拓展性 研究必须的投入产出延长表的编制方法和 意义进行了介绍。其次对灾害冲击下, 造成 相同直接经济损失下的不同经济结构的重 建恢复情况进行模拟; 灾害的恢复期较短情 况下，使用非灾害年数据源时，考虑灾后重 建恢复过程中由于逐年经济结构调整, 对灾 害的间接经济损失的影响。

研究表明同样直接损失, 在不同经济 结构下造成的间接经济损失相差 $13 \%$ 左右， 而恢复期相差不明显, 因此使用接近灾害发 生年的投入产出数据对结果影响有限, 适用 于应急状态下的粗略评估。本文为使用 ARIO 模型进行灾害经济损失风险预测分析 提供了理论依据。

\section{致谢}

本项目得到了 1.河北省自然科学基金青年基金 “区域产业结构调整下的灾害间接经济影响重建 模型研究” (编号：D2015507046)；2.武警学院博 士科研创新项目 “城市灾害链式演化机理及组合干 预研究” 的支持。

\section{参考文献}

[1] S. Hallegatte. An Adaptive Regional Input-Output Model and its Application to the Assessment of the Economic Cost of Katrina. Risk Analysis. 2008, 28(3):779-799.

[2] J.Wu, N. Li, S. Hallegatte, et al. Regional indirect economic impact evaluation of the 2008 Wenchuan Earthauake. Environmental
Earth Science, DOI:

10.1007/s12665-011-1078-9.

[3]丁先军，杨翠红，祝坤福. 基于投入-产 出模型的灾害经济影响评价方法. 自然 灾害学报，2010,19(2)：113-118.

[4]李宁, 吴吉东. 基于 ARI0 模型的汶川地震 灾后恢复重建期模拟. 自然灾害学报, 2012, 21(2) : 68-75.

[5]张鹏，李宁，吴吉东，等，基于 ARI0 模 型的区域洪涝灾害经济影响评价. 长江 流域资源与环境, 2012，21(6)：773-778.

[6]张鹏，李宁，吴吉东. 1998 年湖南洪涝灾 后经济恢复情景模拟, 首届中国沿海地区 灾害风险分析与风险管理学术研讨会, 上 海: 中国灾害防御协会风险分析专业委员 会, 2011：365-371.

[7]张鹏, 李宁. ARI0 模型对灾害引起的部门 经济损失数据完备程度的敏感性分析一 一以湖南为例。灾害学, 2014, 29 (4) : 105-110.

[8]W. Zhang, Y.G. Hu, J.H. Wang, , et al. Research on Urban Waterlogging Disaster Risk Assessment Based on ARCGIS and MIKE FLOOD - A case Study on Shijiazhuang. The Journal of Risk Analysis and Crisis Response, 2015, 5(4):226-233

[9] 廖明球. 投入产出及其扩展分析. 北京： 首都经济贸易大学出版社，2009. 